\title{
Producciones artísticas en el aprendizaje de la enfermería
}

\author{
Montserrat Roca-Roger, Inmaculada Úbeda-Bonet, Carmen Caja-López, Victoria Morín-Fraile, M. Teresa Icart-Isern
}

Objetivo. Facilitar el aprendizaje autónomo y reflexivo del estudiante de enfermería a través del análisis de creaciones artísticas (cine, literatura y artes plásticas).

Sujetos y métodos. En el curso académico 2008-2009 se diseñó una ficha técnica y didáctica para cada obra seleccionada que permite el análisis y la reflexión sobre los procesos de salud-enfermedad. El uso de estas fichas-guía se utilizó en una asignatura troncal y en dos optativas para que fueran analizadas como complemento a los contenidos de las asignaturas. Además, se diseñó una página web en la que aparecen fichas de cine, literatura y artes plásticas.

Resultados. En esta experiencia participaron 340 estudiantes matriculados en la asignatura 'Enfermería comunitaria II', 70 estudiantes de la asignatura 'Atención a la familia en el domicilio' y 30 estudiantes de la asignatura 'La creación artística como soporte en el aprendizaje de la enfermería comunitaria'. Las obras utilizadas con más frecuencia han sido: Te doy mis ojos, Bailar por dentro, Tierras de penumbra (cine); Paula, Diario de una buena vecina, La soledad de los números primos (literatura); Acechando la muerte, La morfina, El grito (artes plásticas).

Conclusiones. Analizar una obra artística ha supuesto para los estudiantes una mayor comprensión de fenómenos que se explican en clase de forma aislada, aunque en la realidad se producen globalmente. Este aspecto se ha comprobado en el rendimiento académico de los estudiantes y en el enriquecimiento de los debates en el aula.

Palabras clave. Aprendizaje. Creaciones artísticas. Enfermería.

\section{Artistic productions in the learning of nursing}

Aim. To facilitate autonomous and reflective learning to nursing students through the analysis of artistic creations (cinema, literature and visual arts).

Subjects and methods. In the academic year 2008-2009 a technical and didactic tab was designed for each selected artistic work allowing the analysis and reflection on the health-disease processes. These tab guides were used in one core and two optional matters to be analyzed as a complement to the course content. In addition, a web page showing chips cinema, literature and visual arts was designed.

Results. This experiment involved 340 students enrolled in the course Community Nursing II, 70 students of the subject Family care at home and 30 enrolled for the course Artistic creation as a learning support in community nursing. The works that were most frequently used were: Take my eyes, Inside I'm dancing, Shadowlands (cinema); Paula, Journal of a good neighbour, The solitude of prime numbers (literature); Stalking death, Morphine, The scream (visual arts).

Conclusions. Analyzing a work of art led the students to greater understanding of phenomena discussed in class as separate items but that in reality are produced as a single whole This was proven in the academic performance of students and enriched discussions in the classroom.

Key words. Artistic creations. Learning. Nursing.

\section{Introducción}

La salud y la enfermedad como realidades humanas han estado y están presentes en las diferentes expresiones artísticas (cine, pintura, escultura, fotografía, literatura, etc.) [1]. El arte refleja situaciones que se dan en la vida real, en el proceso de saludenfermedad, y ayuda a reflexionar sobre ellas. Las creaciones artísticas remiten a historias, épocas, conflictos, costumbres, tratamientos, etc., que ayudan a los estudiantes a entender y analizar aspectos similares a los que se dan en la realidad y con los que tendrán que enfrentarse al acabar su formación y que no siempre encontrarán en los contenidos de las distintas asignaturas que cursan [2].

Las creaciones artísticas, al plasmar situaciones verosímiles, ponen al alumno ante realidades en las que aparecen múltiples matices de las personas y
Escuela de Enfermería. Universitat de Barcelona. L'Hospitalet de Llobregat, Barcelona, España.

Correspondencia:

Dra. Montserrat Roca Roger. Escola d'Infermeria. Universitat de Barcelona. Feixa Llarga, $s / n$.

Pavello de Govern. E-08903 L'Hospitalet de Llobregat (Barcelona).

E-mail:

rocar@ub.edu

Financiación:

Subvención por parte de la Agència de Gestió d'Ajuts Universitaris i de Recerca (AGAUR) de la Generalitat de Catalunya, en la convocatoria para la mejora de la calidad docente de las universidades catalanas del año 2008.

Recibido: 25.06.15.

Aceptado: 28.07.15.

Conflicto de intereses: No declarado.

Competing interests: None declared.

(c) 2016 FEM 
los contextos en que viven. Por ello, tanto el cine, como la literatura y las obras plásticas pueden ser una herramienta útil para ayudar al estudiante a integrar los diversos conocimientos que recibe de forma fragmentada a través de las distintas asignaturas de la disciplina que estudia [3-6]. Además, le permite conocer al sujeto de su atención desde 'otras miradas', como señala Barbado-Hernández refiriéndose a la literatura [7].

Las directrices para las enseñanzas universitarias dentro del Espacio Europeo de Educación Superior hacen especial énfasis en el trabajo que debe realizar el alumno para alcanzar las competencias propias de su grado; esto se ha traducido en la incorporación de metodologías que facilitan el aprendizaje autónomo y la evaluación continuada $[8,9]$.

La utilización del cine, la literatura, la pintura o la obra gráfica en el aprendizaje de ciencias de la salud como la enfermería y la medicina, entre otras, representa un recurso capaz de complementar otros métodos docentes y puede constituir un medio muy valioso para fomentar el aprendizaje centrado en la observación, la reflexión, la investigación y, en general, la resolución de problemas, al mismo tiempo que se brinda a los estudiantes la oportunidad de adquirir una visión más amplia de la cultura $[6,10,11]$.

Además, la reflexión sobre situaciones cotidianas del proceso de salud-enfermedad reflejadas en las obras de arte puede ayudar a la adquisición de habilidades y actitudes, en tanto que coloca al estudiante en situaciones próximas a la realidad, posibilitando que éstos se replanteen las formas de actuar a partir de las reflexiones y el análisis crítico proporcionado por las obras artísticas [12-14].

Con el objetivo de facilitar la integración de los conocimientos que reciben los estudiantes en la Escuela de Enfermería de la Universitat de Barcelona y la realidad que deberán afrontar como profesionales, y con el fin además de fomentar en ellos una actitud reflexiva y crítica, un grupo de profesoras de dicha escuela se planteó desarrollar una experiencia basada en la utilización de diferentes producciones artísticas en el aprendizaje de la enfermería.

\section{Objetivos}

El objetivo general del trabajo ha sido facilitar el aprendizaje autónomo y reflexivo del estudiante de enfermería a través del análisis de creaciones artísticas como instrumento que favorezca la comprensión de situaciones de salud-enfermedad.

Los objetivos específicos fueron:
- Seleccionar diferentes obras artísticas (cine, literatura, artes plásticas) que reflejen situaciones verosímiles relacionadas con el proceso de saludenfermedad-atención como soporte al aprendizaje y a la evaluación continuada del estudiante.

- Crear una página web que contenga un banco de fichas didácticas que sirvan para el análisis de las obras artísticas seleccionadas y su relación con los diferentes contenidos curriculares del Grado de Enfermería, y poner esta página web a disposición de la comunidad educativa.

- Instaurar un espacio de reflexión que permita a los estudiantes la integración de conocimientos de las diferentes materias y asignaturas que configuran sus estudios.

- Facilitar a los estudiantes una experiencia de aprendizaje diferente y motivadora en su área de conocimiento y la apertura a otras disciplinas, además de promover en ellos un saber sobre el arte.

\section{Metodología y plan de trabajo}

\section{Identificación de materiales}

Para conocer si se utilizaba el cine, la literatura o las artes plásticas como soporte a la docencia se envió a las diferentes escuelas de enfermería de España, a través de correo electrónico, un cuestionario en el que se preguntaba si utilizaban algún tipo de material artístico de libre disposición. Si la respuesta era afirmativa, tenía que identificarse el tipo de material (soporte y título de la obra), en qué asignatura se utilizaba y si existía alguna guía para su aplicación.

De los cuestionarios recibidos, de entre los 40 enviados, 23 escuelas respondieron afirmativamente. El recurso que más se recomendaba era el cine, seguido de la novela. La asignatura que más empleaba el cine era enfermería psiquiátrica y psicosocial. Ninguna escuela tenía una guía estandarizada para su uso.

A continuación, el equipo investigador visualizó y analizó las obras indicadas por las escuelas consultadas y también se realizó una búsqueda bibliográfica sobre experiencias previas y sobre páginas web de cine, literatura y artes plásticas [15-18]. Además, se tuvieron en cuenta aquellas obras que las autoras habían leído o visualizado en los dos últimos años y que consideraron adecuadas para alcanzar los objetivos del trabajo. Una vez identificadas las nuevas obras, se analizó la pertinencia de su contenido para utilizarlas en la docencia.

Los requisitos para la selección de las diversas obras fueron que su temática estuviera relacionada 
con el ámbito de la salud-enfermedad-atención, que resultaran atractivas para los estudiantes y de fácil acceso, y que no contravinieran ninguna ley. Con todo este material se configuró una nueva asignatura optativa de tres créditos impartida en el cuarto semestre de la titulación de enfermería. Durante su impartición se pidió a los estudiantes que identificaran una nueva obra de cada uno de los tres géneros (cine, literatura y obra plástica). Estas obras fueron también analizadas por las profesoras y aquellas que consideraron pertinentes también se incluyeron en el catálogo.

\section{Preparación de las fichas}

Se diseñó una ficha para cada obra seleccionada que contiene los datos técnicos sobre ella y una guía didáctica que permite el análisis y la reflexión sobre los procesos de salud-enfermedad-atención.

La guía didáctica contiene los siguientes apartados que pretenden orientar el aprendizaje del estudiante:

- Área temática donde adscribirla y aspectos que trata teniendo como referencia las asignaturas de la carrera de enfermería.

- Objetivos de aprendizaje.

- Contenidos que refuerza.

- Fragmentos de interés.

- Preguntas para analizar y fijar conceptos. Dichas preguntas hacen que el estudiante deba buscar información en diferentes fuentes para conseguir los objetivos de aprendizaje propuestos en cada obra seleccionada.

Se ha construido un banco con las fichas de las obras seleccionadas. En las figuras 1, 2 y 3 aparecen tres ejemplos de esas fichas.

\section{Prueba piloto para valorar la experiencia}

Antes de iniciar el curso académico 2008-2009 se elaboró un primer banco de fichas sobre producciones artísticas para que se pudiera incluir en la Intraned de las diversas asignaturas a través de la plataforma Moodle. En estas fichas se daba un enlace para facilitar la obtención de la obra mencionada, en artes plásticas, que correspondía a las páginas web de los museos donde estaban expuestas. Para las obras literarias y películas, los estudiantes debían acudir a bibliotecas y videotecas para obtenerlas en préstamo, o bien adquirirlas.

Del banco de fichas elaboradas sobre creación artística, se seleccionaron aquellas obras que se con-
Figura 1. Película: A propósito de Henry.

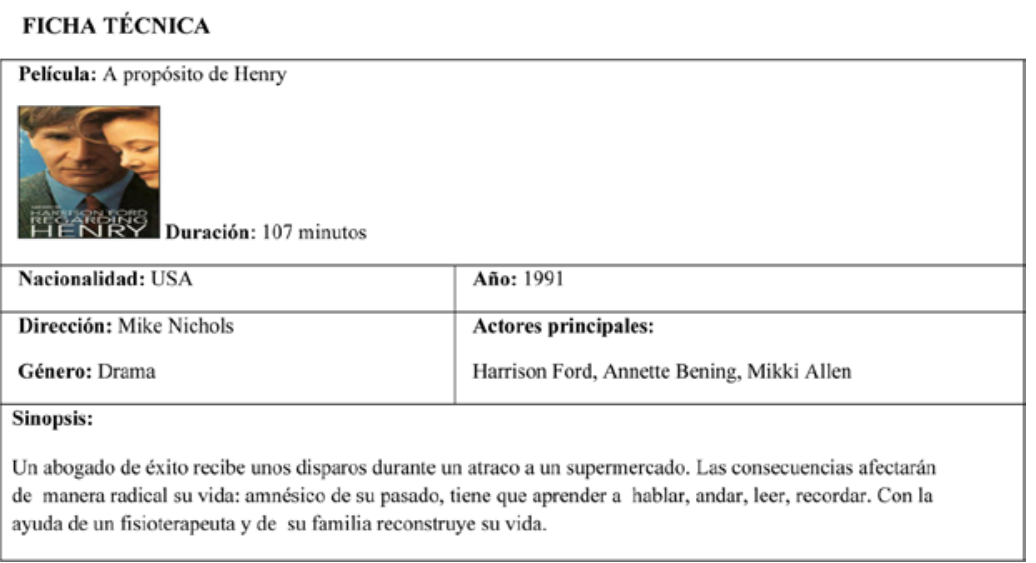

\section{FICHA DIDÁCTICA}

\begin{tabular}{l} 
Película: A propósito de Henry \\
\hline Área temática donde adseribirla: Enfermeria Clínica, Fundamentos de Enfermería. \\
Aspectos que trata: Sistema familiar. Cuidados familiares. Profesionalismo. \\
Objetivos de aprendizaje: \\
Identificar los cuidados que requiere una persona con afectaciones medulares. \\
Analizar las consecuencias derivadas de una afectación medular en la satisfacción de las necesidades básicas. \\
Identificar el impacto supone el hecho que el padre de familia sufra una afectación medular. \\
Valorar la importancia de la autosuperación y del papel de la familia en el afrontamiento del problema. \\
Contenidos teóricos que apoya la película: \\
Lesiones medulares: tipologia, evolución y cuidados. \\
Afectación de las necesidades básicas de las personas con lesión medular. \\
Aprendizaje de habilidades perdidas \\
Fragmentos o secuencies de interés (situarlo en el tiempo transeurrido desde el inicio): \\
A lo largo de toda la pelicula. \\
Aspectos que hace falta clarificar: \\
Preguntas para resolver y fijar conceptos: \\
¿Qué cuidados de enfermería requiere una persona con una lesión medular similar a la que aparece en la película, \\
en las diferentes etapas de su proceso de recuperación? \\
¿Cuál debería ser el papel de la familia con un miembro afectado por una lesión medular? \\
¿Cuáles son los principales aprendizajes a los que se debe enfrentar una persona con una lesión medular? \\
Documentación utilizada: http://www.filmaffinity.com/es/film938141.html
\end{tabular}

sideraron pertinentes para la asignatura troncal 'Enfermería comunitaria' y para la asignatura optativa 'Atención a la familia en el domicilio'. Se recomendó al alumnado que escogiera una obra literaria, una película y una obra de artes plásticas para que fuera analizada como complemento a los contenidos de las asignaturas. En la asignatura troncal, los alumnos, dependiendo de la obra escogida, tenían la posibilidad de profundizar en los sistemas sanitarios, la salud pública, el maltrato, las desigualdades sociales relacionadas con la salud, las discapacidades, etc. En la asignatura optativa se hizo es- 
Figura 2. Obra literaria: Tiempo de silencio.

\section{FICHA TÉCNICA}

\begin{tabular}{|c|c|}
\hline Obra literaria: Tiempo de s & \\
\hline Nacionalidad: española & Año: primera edición 1962 \\
\hline $\begin{array}{l}\text { Autor: Luís Martin-Santos } \\
\text { Editorial: Seix Barral } \\
\text { Género: Novela }\end{array}$ & $\begin{array}{l}\text { Traducción: } \\
\text { Título original: }\end{array}$ \\
\hline Sinopsis: & \\
\hline $\begin{array}{l}\text { Pedro es un joven médico ir } \\
\text { sobre el cáncer se realizan } \\
\text { laboratorio. Un trabajador d } \\
\text { ratones tan dificiles de obten } \\
\text { de los pechos de Florita, un } \\
\text { investigador y su aceptación } \\
\text { económico de los años } 60 \text { y } 1\end{array}$ & $\begin{array}{l}\text { de finales de los años } 50 \text {. Sus investigaciones } \\
\text { nado de ratones, que no se reproducen en el } \\
\text { Muecas, se encarga de proporcionarle estos } \\
\text { o que críen a su choza gracias al calor corporal } \\
\text { lente provoca la expulsión de Pedro del centro } \\
\text { cio que supuso la España anterior al desarrollo }\end{array}$ \\
\hline
\end{tabular}

\section{FICHA DIDÁCTICA}

Obra: Tiempo de silencio

\section{Área temática donde adscribirla:}

Enfermería quirúrgica, Enfermería de salud comunitaria, Ética en enfermería.

\section{Objetivos de aprendizaje:}

Relacionar las desigualdades sociales con las desigualdades en salud.

Identificar los posibles riesgos para la salud de las personas que viven en situación de marginalidad

Conocer las actuaciones de la enfermería comunitaria con los sectores sociales desfavorecidos.

Conocer la legislación referente al aborto.

Citar actividades preventivas de embarazos no deseados

Contenidos teóricos que apoya

Sistema sanitario; Investigación; Salubridad; Salud sexual y reproductiva; Adicciones

Fragmentos de interés (situarlos en el capítulo al que pertenecen):

Aspectos que hace falta clarificar:

Preguntas para resolver $y$ fijar conceptos:

¿Cuáles son las actividades de la enfermera comunitaria en la prevención de embarazos no deseados? ¿Qué dice la legislación española sobre el aborto?

¿Existen diferencias entre los diferentes países europeos en dicha legislación?

Cite cinco situaciones de desigualdades sociales actuales y que podrian comportar desigualdades en

salud. ¿Qué se debería hacer desde la salud comunitaria para evitar que estas desigualdades se conviertan en desigualdades en salud?

\section{Documentación utilizada:}

http://es.wikipedia.org/wiki/Tiempo_de_silencio_(novela)

http://www.fisterra.com/human/1libros/ficcion/tiempo_silencio.asp

http://www.librosgratisweb.com/html/santos-luis-martin/tiempo-de-silencio/index.htm pecial énfasis en la carga de los cuidadores familiares, los cuidados enfermeros en el domicilio, las pérdidas y el duelo.

Ese mismo año se empezó a impartir la asignatura optativa 'La creación artística como soporte en el aprendizaje de la enfermería comunitaria'. Su objetivo era utilizar la creación artística como vehículo para que el alumnado analizara y discutiera situaciones verosímiles del proceso de salud-enfermedad-cuidados. En ella, los estudiantes analizaban, discutían y podían profundizar, a partir de la lectura y visualización de diferentes obras artísticas, aspectos relacionados con la salud, la enfermedad, el cuidado de las personas, las relaciones interpersonales, etc. Esta asignatura se impartió a estudiantes en su último año, por lo que sirvió para realizar una síntesis de los diferentes contenidos.

Al inicio de la asignatura, las profesoras clarificaban el trabajo que debía realizar cada estudiante. Este trabajo consistía en la selección, a partir del banco de fichas disponibles en la intranet, de una obra de cada uno de los tres géneros artísticos propuestos y, con la ayuda de la guía de cada ficha, realizaban su análisis, que debía ser entregado a través de la plataforma Moodle. Al final de cada género analizado, los estudiantes exponían su análisis en el aula, que era discutido por todo el grupo-clase. Así pues, cada estudiante analizaba una obra literaria, una película y una obra de artes plásticas, exponía su análisis públicamente y discutía los hallazgos con todos sus compañeros.

En esta experiencia piloto participaron 340 estudiantes matriculados en la asignatura 'Enfermería comunitaria II', 70 estudiantes de la asignatura 'Atención a la familia en el domicilio' y 30 de la asignatura 'La creación artística como a soporte en el aprendizaje de la enfermería comunitaria'.

Al finalizar el curso se solicitó la opinión de los estudiantes con relación a la experiencia. A continuación se transcriben algunas de sus opiniones: 'me ha ayudado a relacionar lo que se explica en varias asignaturas', 'creo que he visto cosas con las que me encontraré como enfermera,' 'me ha generado el hábito de buscar cosas de enfermería cuando hago otra actividad, 'me hubiera gustado hacer más, me llevo la lista para el verano', 'me ha supuesto tener que leer más libros, con lo que no contaba'.

\section{Aplicación de la metodología en otros cursos académicos}

Debido a la buena acogida y a los resultados académicos obtenidos, se creyó conveniente seguir con la 
experiencia de introducir en las asignaturas la recomendación de utilizar creaciones artísticas al mismo nivel que la habitual bibliografía recomendada. Esta experiencia ha continuado hasta el curso 20122013.

La modificación más importante que se realizó en los contenidos de las asignaturas 'Enfermería comunitaria' y 'Atención a la familia en el domicilio' fue que el profesorado seleccionó las obras que los estudiantes debían analizar.

La asignatura optativa 'La creación artística como soporte en el aprendizaje de la enfermería comunitaria' ha continuado impartiéndose de la misma forma excepto que, por normativa de la Escuela de Enfermería, se imparte durante el primer curso del grado. Ello ha supuesto que esta asignatura permita acercar al alumnado a situaciones verosímiles cuando aún desconoce, en gran medida, qué supone convivir con la enfermedad y las pérdidas; además, les permite anticipar situaciones globales relacionadas con estos aspectos que, tal vez, no podrían identificar de otra manera.

Además, también través de la intranet de la Universitat de Barcelona (plataforma virtual Moodle), se ha puesto a disposición de la comunidad educativa una página web con las fichas didácticas de las obras seleccionadas. En dicha página aparecen fichas de cine y literatura de fácil adquisición y sobre obras plásticas expuestas en diversos museos (actualmente disponible en www.ub.edu/cinemaisalut). Las obras utilizadas con mayor frecuencia han sido:

- Cine: Te doy mis ojos (I. Bollain, 2003), Bailar por dentro (D. O’Donell, 2004), Tierras de penumbra (R. Attenborough, 1993).

- Literatura: Paula (I. Allende, 1985), Diario de una buena vecina (D. Lessing, 1983), La soledad de los números primos (P. Giordano, 1982).

- Artes plásticas: Acechando la muerte (K. Carter, 1994), La morfina (S. Rusiñol, 1894), El grito (E. Munch, 1893).

Actualmente se han elaborado 46 fichas sobre cine, 14 sobre literatura y 53 sobre artes plásticas. En la página web mencionada están introducidas para su consulta 46 fichas sobre películas, 13 sobre literatura y 16 sobre artes plásticas; el resto está en revisión.

En conclusión, analizar una obra artística relacionada con el proceso salud-enfermedad-atención siguiendo la guía didáctica, ha supuesto para los estudiantes:

- Una mayor comprensión de fenómenos que se explican en clase de forma aislada, aunque en la
Figura 3. Pintura: La morfina.

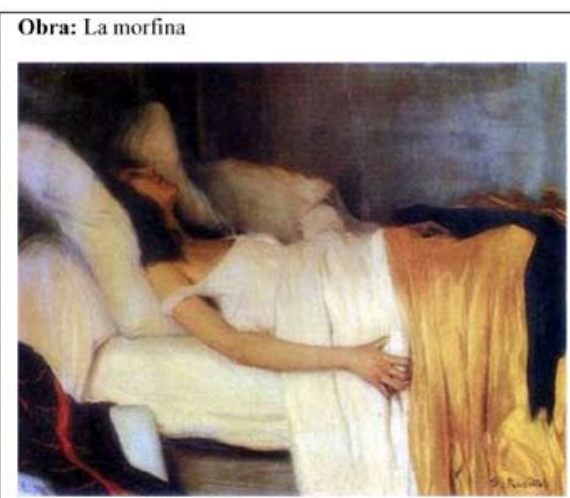

Dimensiones: $87,36 \times 115 \mathrm{~cm}$

\begin{tabular}{l|l|}
\hline Nacionalidad: española (catalana) & Año: 1894 \\
\hline $\begin{array}{l}\text { Autor: Santiago Rusiñol i Prats } \\
\text { Técnica: óleo sobre tela }\end{array}$ & $\begin{array}{l}\text { Época-corriente: s. XVIII-XIX } \\
\text { Localización: Cau Ferrat de Sitges }\end{array}$ \\
\hline $\begin{array}{l}\text { Descripción: } \\
\text { El cuadro muestra una joven en la cama bajo los efectos de la morfina. La figura de la joven, en tensión, } \\
\text { por una posible convulsión, presenta un juego cromático interesante, entre los ocres, negros y blancos. } \\
\text { Contrasta la delicadeza de la pintura y la inquietud que provoca la escena. La chica agarra con las } \\
\text { sábanas, mientras su cara muestra serenidad. }\end{array}$
\end{tabular}

\section{FICHA DIDÁCTICA}

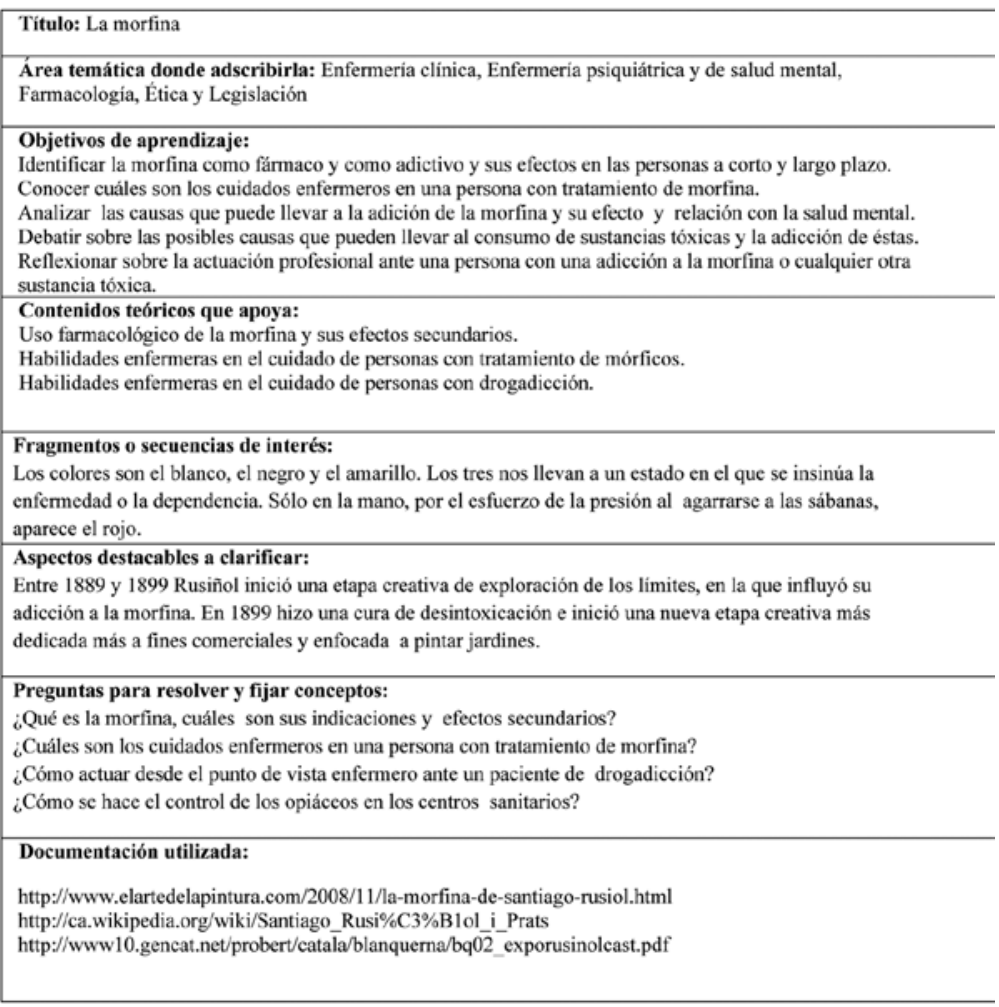


realidad se producen globalmente. Este aspecto se ha comprobado en el rendimiento académico de los estudiantes.

- El enriquecimiento de los debates en clase al tener todos los estudiantes los mismos referentes para la discusión.

- Una ayuda para relacionar la teoría con la realidad y desarrollar una actitud crítica ante situaciones de cuidados.

- La posibilidad de familiarizarse y analizar materiales que de otra forma, quizás, no hubieran conocido.

- Una forma novedosa y lúdica de aprendizaje.

Para las profesoras implicadas en el proyecto, el análisis de creaciones artísticas ha representado un complemento a la docencia de las ciencias de la salud. Esta experiencia ha puesto de manifiesto que la incorporación del cine, la literatura o la obra plástica es posible como una asignatura optativa o como un elemento complementario dentro de otras asignaturas.

En nuestra opinión, la novedad de la experiencia narrada reside en la variedad de materiales utilizados para promover el aprendizaje de los estudiantes $y$ en ofrecer una herramienta a la comunidad docente, como es la página web, que presenta diferentes obras para analizar con una guía que incluye sugerencias para su utilización.

\section{Bibliografía}

1. García-Sánchez JE, Trujillano-Martín I, García-Sánchez E. Medicina y cine. ¿Por qué? Revista de Medicina y Cine 2005; 1 URL: http://www.usal.es/ revistamedicinacine/Indice_2005/ Revista/numero_1/version_espanol/esp_1/ed_esp_1.pdf. [13.05.2013].
2. Rabuzzi KA, ed. Toward a new discipline. Lit Med 1982; 1: 1-118.

3. Fresnadillo MJ, Diego C, García E, García JE. Metodología docente para la utilización del cine en la enseñanza de la microbiología médica y las enfermedades infecciosas. Revista de Medicina y Cine 2005; 1. URL: http://campus. usal.es/ revistamedicinacine/Volumen_2_1/n4/esp_4_htlm/ editorial2_4.htm. [20.04.2014].

4. De Pablos-Pons J. Cine didáctico. Posibilidades y metodología. Madrid: Narcea; 1980.

5. Hunter KM, Charon R, Coulehan JL. The study of literature in medical education. Acad Med 1995; 70: 787-94.

6. Baños JE. El valor de la literatura en la formación de los estudiantes de medicina. Educ Med 2003; 6: 93-9.

7. Barbado-Hernández FJ. Medicina y literatura en la formación del médico residente de medicina interna. Ann Med Int 2007; 24: 195-200.

8. Brown S. Estrategias institucionales en evaluación. In Brown S, Glasner A, eds. Evaluar en la universidad. Problemas y nuevos enfoques. Madrid: Narcea; 2003. p. 23-33.

9. Rico-Vercher M, Rico-Pérez C. El portfolio discente. Alcoy: Marfil; 2004.

10. Baños JE, Aramburu JF, Sentí M, Biocinema: la experiencia de emplear películas comerciales con estudiantes de biología. Revista de Medicina y Cine 2005; 1. URL: http://campus. usal.es/ revistamedicinacine/numero\%202/esp_2/biocinema esp.pdf. [18.06.2013].

11. Álvarez-Díaz JA. Importancia de la literatura dentro de las humanidades médicas. Gac Med Mex 2010; 146: 71-5.

12. Cuesta-Benjumea C. La investigación cualitativa y el desarrollo del conocimiento en enfermería. Texto contexto - enferm 2010 19: 762-6.

13. Loscos J, Baños JE, Loscos F, De la Cámara J. Medicina, cine y literatura: una experiencia docente en la Universitat Autònoma de Barcelona. Rev Med Cine 2006; 2: 138-42.

14. Craice de Benedetto MA, Gatti G, Lima da Costa D. La literatura como recurso didáctico en la formación humanística de los estudiantes de medicina. Aten Fam 2011; 18: 59-62.

15. Internet Movie Database (IMDb). URL: http://www.imdb.com [12.04.2009].

16. Charon R. Reading, writing, and doctoring: literature and medicine. Am J Med Sci 2000; 319: 285-91.

17. Suarez-Santos M. Arte y medicina. La enfermedad a través de la pintura (2009). URL: http://huidadelmundanalruido. zoomblog.com/archivo/2009/05/14/arte-Y-Medicina-LaEnfermedad-A-Traves.html. [10.08.2009].

18. Ciudad de la pintura. Madrid: Asociación Ayúdale a caminar. URL: http://pintura.aut.org. [25.02.2009]. 